\title{
Estudo do perfil lipídico de crianças e jovens até 19 anos de idade
}

\author{
Lipidic profile study in children and youngsters up to 19 years old
}

\author{
Mario Seki \\ Marisa Okabe Seki \\ Adriano Damasceno Lima ${ }^{3}$ \\ Miltes Hitomi Oba Onishi ${ }^{4}$ \\ Matiko Okabe Seki ${ }^{4}$ \\ Leandro Augusto Granja de Oliveira ${ }^{2}$
}

\begin{tabular}{|c|c|}
\hline unitermos & resumo \\
\hline $\begin{array}{l}\text { Dislipidemias } \\
\text { Doenças ateroscleróticas } \\
\text { Hipercolesterolemia } \\
\text { Hipertrigliceridemia } \\
\text { Hiperlipidemia }\end{array}$ & $\begin{array}{l}\text { Foi realizado levantamento retrospectivo de } 624 \text { casos, de ambos os sexos, com idades } \\
\text { variando de } 3 \text { a } 19 \text { anos, que realizaram exames do perfil lipídico (PL) em laboratório privado } \\
\text { de Londrina-PR. A prevalência das dislipidemias - adotando os valores de referência } \\
\text { estabelecidos pelo Segundo Consenso Brasileiro sobre Dislipidemias (SCBD) - é alarmante na } \\
\text { casuística estudada. As autoridades necessitam estender a investigação aos usuários do } \\
\text { sistema público de saúde para avaliar a dimensão real do problema na população geral. }\end{array}$ \\
\hline
\end{tabular}

\section{abstract}

This retrospective survey of 624 cases with lipidic profile used subjects of both sexes until 19 years old. These tests were conducted in private laboratory in Londrina, Paraná, Brazil. In the studied cases, the results, according to the reference values established by Segundo Consenso Brasileiro sobre Dislipidemias, showed a high prevalence of dyslipidemia. These results also show that in order to evaluate the real dimension of the problem, it is necessary to include the public health system in the experience. key words

Dyslipidemia

Atherosclerotic disease

Hypercholesterolemia

Hypertriglyceridemia

Hyperlipidemia

\section{Introdução}

As dislipidemias estão, indissoluvelmente, vinculadas a fatores de risco das doenças ateroscleróticas (DAs) - que se encontram entre as principais causas de morbidade e mortalidade em adultos $(4,7)$. Os fatores de risco das DAs têm início na infância e tendem a persistir na vida adulta $(4,6,7)$. O Segundo Consenso Brasileiro sobre Dislipidemias (SCBD) recomendou rastreamento seletivo do perfil lipídico (PL) em crianças e jovens até 19 anos, incluindo cuidados com coleta de material, valores de referência, controle de qualidade, confirmação de resultados limítrofes ou alterados e tratamentos $(8,9)$. Apesar da extensa literatura internacional abordando dislipidemias em crianças e jovens, não foram observadas publicações recentes sobre sua prevalência em nosso meio.

\section{Objetivo}

O objetivo deste trabalho foi avaliar a prevalência das dislipidemias através de estudo retrospectivo dos exames do PL em um laboratório de análises clínicas, abrangendo casos de ambos os sexos e idades até 19 anos, e classificar os casos alterados conforme as quatro situações definidas pelo SCBD.

\section{Material e método}

\section{Laboratório}

Os dados foram coletados no sistema de informática do Labmed - Laboratório Médico de Lon-
1. Médico patologista clínico. 2. Acadêmico de Medicina da Universidade de Alfenas-MC 3. Acadêmico de Medicina da Universidade Estadual de Londrina-PR. 4. Farmacêutica-bioquímica. 
drina/Labimagem - Diagnóstico Avançado em Medicina, em Londrina-PR.

\section{Casuística}

O levantamento retrospectivo, que abrangeu o período de julho de 2000 a março de 2001, compreendeu casos provenientes de consultórios e clínicas privadas. No total, foram avaliados 624 casos, de ambos os sexos, com idades de 3 a 19 anos, dos quais 483 contendo todos os quatro exames que constituem o perfil lipídico. Não foram apreciados exames de repetição (monitorização terapêutica ou confirmação de resultados anteriores) realizados nesse intervalo de tempo.

\section{Exames}

Analisaram-se resultados de dosagens por reações enzimáticas de colesterol total (CT), triglicerídeos (TG) e colesterol HDL, utilizando-se metodologia de química seca com kits Johnson \& Johnson e aparelho Vitros 750. O colesterol LDL foi obtido através de cálculo de Friedewald para TG abaixo de 400mg/dl; resultados com valores superiores foram quantificados, com determinação espectrofotométrica, utilizando-se kits Merck.

\section{Controle e sistema de qualidade}

O laboratório envolvido neste estudo realiza controle interno e externo de qualidade - Programa de Excelência para Laboratórios Médicos (PELM) -, no qual vem obtendo ótimos desempenhos, ininterruptamente, desde o ano de 1979. Mantém, ainda, sistema de qualidade certificado pela ISO 9002 desde 1999.

\section{Distribuição dos casos e cálculo percentual}

Para distribuição dos casos em resultados desejáveis, limítrofes (para CT e LDL) e alterados, foram adotados valores de referência do SCBD. Os resultados percentuais, conforme sexo e idade $(<10$ e $>10$ anos), foram calculados em relação ao número total de casos de cada grupo.

\section{Classificação das dislipidemias}

A classificação das dislipidemias, nas situações estabelecidas pelo SCBD, considerou os casos que apresentaram todos os quatro exames do PL.

\section{Resultados}

A distribuição dos 624 casos, conforme os resultados obtidos, está na Tabela 1. As Tabelas 2 a 5 revelam a distribuição dos casos conforme os resultados de TG (610), CT (612), LDL (514) e HDL (521). O estudo da classificação identificou e distribuiu 183 casos conforme as situações das dislipidemias (Tabela 6).

\section{Discussão}

Presença de estrias e lesões de gorduras nas aortas e coronárias de crianças e adultos jovens, que morreram por traumas agudos, detalhadas nas investigações do Pathobiological Determinants of Atherosclerosis in Youth (PDAY) e do Bogalusa Heart Study, está relatada em textos de revisão $(6,7,11)$. Dosagens de colesteróis LDL e VLDL em materiais colhidos postumamente revelaram associação direta com acha-

\begin{tabular}{|c|c|c|c|c|c|c|c|}
\hline \multirow{3}{*}{ Grupos } & \multicolumn{7}{|c|}{ Resultados } \\
\hline & \multicolumn{2}{|c|}{ Desejáveis } & \multicolumn{2}{|c|}{ Limitrofes } & \multicolumn{2}{|c|}{ Alterados } & \multirow[t]{2}{*}{ Total de casos } \\
\hline & casos & $(\%)$ & casos & $(\%)$ & casos & $(\%)$ & \\
\hline Masculino & 113 & $(37,5)$ & 67 & $(22,3)$ & 121 & $(40,2)$ & 301 \\
\hline Feminino & 141 & $(43,6)$ & 61 & $(18,9)$ & 121 & $(37,5)$ & 323 \\
\hline Total & 254 & $(40,7)$ & 128 & $(20,5)$ & 242 & $(38,8)$ & 624 \\
\hline$<10$ anos & 218 & $(45,5)$ & 101 & $(21,1)$ & 160 & $(33,4)$ & 479 \\
\hline$>10$ anos & 36 & $(24,8)$ & 27 & $(18,6)$ & 82 & $(56,6)$ & 145 \\
\hline Total & 254 & $(40,7)$ & 128 & $(20,5)$ & 242 & $(38,8)$ & 624 \\
\hline
\end{tabular}




\section{Tabela 2 Triglicerídeos}

\begin{tabular}{l|ccccc}
\hline \multirow{2}{*}{ Grupos } & \multicolumn{3}{|c}{ Desejáveis } & \multicolumn{2}{c}{ Resultados } \\
& casos & $(\%)$ & casos & $(\%)$ & Total de casos \\
\hline Masculino & 223 & $(76,4)$ & 69 & $(23,6)$ & 292 \\
Feminino & 250 & $(78,6)$ & 68 & $(21,4)$ & 318 \\
\hline Total & 473 & $(77,5)$ & 137 & $(22,5)$ & 610 \\
\hline$<10$ anos & 88 & $(62,9)$ & 52 & $(37,1)$ & 140 \\
$>10$ anos & 385 & $(81,9)$ & 85 & $(18,1)$ & 470 \\
\hline Total & 473 & $(77,5)$ & 137 & $(22,5)$ & 610 \\
\hline
\end{tabular}

Tabela 3 Colesterol total

\begin{tabular}{|c|c|c|c|c|c|c|c|}
\hline \multirow{3}{*}{ Grupos } & \multicolumn{7}{|c|}{ Resultados } \\
\hline & \multicolumn{2}{|c|}{ Desejáveis } & \multicolumn{2}{|c|}{ Limitrofes } & \multicolumn{2}{|c|}{ Alterados } & \multirow{2}{*}{ Total de casos } \\
\hline & casos & $(\%)$ & casos & $(\%)$ & casos & $(\%)$ & \\
\hline Masculino & 157 & $(53,4)$ & 100 & (34) & 37 & $(12,6)$ & 294 \\
\hline Feminino & 179 & $(56,3)$ & 96 & $(30,2)$ & 43 & $(13,5)$ & 318 \\
\hline Total & 336 & $(54,9)$ & 196 & (32) & 80 & $(13,1)$ & 612 \\
\hline$<10$ anos & 72 & $(50,3)$ & 54 & $(37,8)$ & 17 & $(11,9)$ & 143 \\
\hline$>10$ anos & 264 & $(56,3)$ & 142 & $(30,3)$ & 63 & $(13,4)$ & 469 \\
\hline Total & 336 & $(54,9)$ & 196 & (32) & 80 & $(13,1)$ & 612 \\
\hline
\end{tabular}

\section{Tabela 4 Colesterol LDL}

\begin{tabular}{|c|c|c|c|c|c|c|c|}
\hline \multirow{3}{*}{ Grupos } & \multicolumn{7}{|c|}{ Resultados } \\
\hline & \multicolumn{2}{|c|}{ Desejáveis } & \multicolumn{2}{|c|}{ Limitrofes } & \multicolumn{2}{|c|}{ Alterados } & \multirow[t]{2}{*}{ Total de casos } \\
\hline & casos & $(\%)$ & casos & $(\%)$ & casos & $(\%)$ & \\
\hline Masculino & 153 & (64) & 51 & $(21,3)$ & 35 & $(14,7)$ & 239 \\
\hline Feminino & 175 & $(63,6)$ & 63 & $(22,9)$ & 37 & $(13,5)$ & 275 \\
\hline Total & 328 & $(63,8)$ & 114 & $(22,2)$ & 72 & $(14)$ & 514 \\
\hline$<10$ anos & 77 & $(65,8)$ & 23 & $(19,7)$ & 17 & $(14,5)$ & 117 \\
\hline$>10$ anos & 251 & $(63,2)$ & 91 & $(22,9)$ & 55 & $(13,9)$ & 397 \\
\hline Total & 328 & $(63,8)$ & 114 & $(22,2)$ & 72 & $(14)$ & 514 \\
\hline
\end{tabular}




\begin{tabular}{|c|c|c|c|c|c|}
\hline \multirow[t]{2}{*}{ Grupos } & \multicolumn{4}{|c|}{$\begin{array}{l}\text { Resultados } \\
\text { Alterados }\end{array}$} & \multirow[t]{2}{*}{ Total de casos } \\
\hline & casos & $(\%)$ & casos & $(\%)$ & \\
\hline Masculino & 189 & $(77,5)$ & 55 & $(22,5)$ & 244 \\
\hline Feminino & 232 & $(83,8)$ & 45 & $(16,2)$ & 277 \\
\hline Total & 421 & $(80,8)$ & 100 & $(19,2)$ & 521 \\
\hline$<10$ anos & 72 & $(63,2)$ & 42 & $(36,8)$ & 114 \\
\hline$>10$ anos & 349 & $(85,7)$ & 58 & $(14,3)$ & 407 \\
\hline Total & 421 & $(80,8)$ & 100 & $(19,2)$ & 521 \\
\hline
\end{tabular}

\section{Tabela 6 Classificação das dislipidemias}

\begin{tabular}{lcc} 
Situações definidas pelo SCBD & Casos & $\%$ \\
TG isolado e elevado & 38 & 20,8 \\
CT isolado e elevado & 35 & 19,1 \\
CT elevado + TG elevado (hiperlipidemia mista) & 19 & 10,4 \\
HDL diminuído isolado ou associado a LDL elevado e/ou TG elevado & 91 & 49,7 \\
\hline Total & 183 & 100 \\
\hline
\end{tabular}

dos vasculares e relação inversa com o nível de HDL $(6,7)$.

A avaliação da Tabela 1 demonstra que $38,8 \%$ do total dos casos apresentaram resultados alterados, com predomínio no grupo etário > 10 anos (56,6\%) e tendência de equilíbrio entre os sexos. Observa-se também que $20,5 \%$ do total dos casos encontram-se na faixa limítrofe para CT e LDL. Trata-se de dado alarmante diante das evidências das vinculações das dislipidemias com as DAs citadas neste estudo. Os dados de TG (Tabela 2) mostram resultados alterados com predomínio do grupo $<10$ anos $(37,1 \%)$ e tendência de equilíbrio entre os sexos. Resultados de CT (Tabela 3) e LDL (Tabela 4) demonstram resultados alterados com tendência de equilíbrio em relação a sexo e idade. Os resultados de HDL isolado (Tabela 5) evidenciam predomínio de casos alterados no grupo masculino (22,5\%) e na faixa etária < 10 anos (36,8\%).

A classificação das situações dos 183 casos de dislipidemias (Tabela 6) revela predomínio de HDL diminuído, isolado ou associado às elevações de LDL e/ ou TG (49,7\%). Essa associação tende a ser mais aterogênica (1) e, ao lado do tabagismo, está relacionada com precoce estágio de aterosclerose em jovens (6).

Para avaliar a extensão da dislipidemia nesse grupo etário na população geral, diante da procedência dos casos, há necessidade de as autoridades públicas investigarem se as tendências encontradas neste trabalho se aplicam aos usuários de serviços públicos de saúde. Os profissionais da medicina laboratorial podem contribuir para a saúde da população realizando avaliações regionais de prevalência das dislipidemias. Confirmando-se as tendências apresentadas neste estudo, haverá necessidade de alertar e mobilizar a comunidade e as organizações de saúde sobre os riscos futuros, além de estimular programas de prevenção das doenças cardiovasculares nas escolas dirigidos a crianças e adolescentes em todos os níveis, como propõe Berenson (2).

Os valores de referência aplicados neste estudo, estabelecidos pelo SCBD para CT, LDL e HDL, foram transcritos do National Cholesterol Education Program (NCEP, 1993), e os de TG foram baseados nas determinações da Sociedade Européia de Aterosclerose. 
Considerando-se as diferenças em relação aos hábitos alimentares da população brasileira, há necessidade de se estabelecer parâmetros locais, pois esses valores podem não refletir a realidade nacional. Estudos adicionais em nosso meio são necessários para correlacionar as diferentes situações das dislipidemias com influências de hereditariedade (6), características raciais $(1,2)$, obesidade $(2,6)$, dieta alimentar $(5)$, uso de medicamentos $(6,9)$ e tabagismo $(6,11)$.

Para efeito de diagnóstico e tratamento, os casos encontrados com valores alterados merecem criteriosa análise individual, repetindo-se os exames no mínimo duas vezes, com intervalo de duas semanas, conforme recomendações do SCBD (8). Cuidados específicos para tratamento das dislipidemias em crianças e adultos jovens estão bem estabelecidos $(3,7,10)$.

\section{Conclusões}

Os dados obtidos neste levantamento revelam ocorrência de dislipidemias em $38,8 \%$ do total dos casos estudados, e $20,5 \%$ com resultados limítrofes para CT e LDL. A classificação das situações, adotando-se padronização do SCBD, apresenta, nesta casuística, predomínio do HDL diminuído, isolado ou associado a elevações de TG e/ou LDL (49,7\%).

\section{Agradecimentos}

Agradecemos ao dr. Pedro Henrique Godinho, ao dr. Luis Parellada Ruiz, a Kazuhiro Ito, ao dr. Eduardo Andreghetti, a Eduardo Garcia da Silveira Filho, a Sandra Albertini e a Rozilda Lemos o apoio e as sugestões.

\section{Referências}

1. Andriolo, A. Lipídeos. In: Carraza, F.R. \& Andriolo, A. Diagnóstico laboratorial em pediatria. 1.ed. São Paulo: Sarvier, 2000. p. 99-104.

2. Berenson, G.S. Prevention of heart disease beginning in childhood through comprehensive school health: the heart smart program. Prev. Med., 22: 507-13, 1993.

3. Diament, J. et al. Crianças, mulheres, idosos, pacientes submetidos a transplante e renais crônicos: estratégias e precauções na terapêutica das dislipidemias. Rev. Soc. Cardiol. Estado de São Paulo, 10: 802-3, 2000.

4. Komatsu, A.; Sakurai, I. \& PDAY Research Group. A study of the development of atherosclerosis in childhood and young adults: risk factors and the prevention of progression in Japan and the USA. Pathol. Intern., 46: 541-7, 1996.

5. Lapinteimu, H. et al. Prospective randomised trial in 1062 infants of dietlow in saturad fat and cholesterol. Lancet, 345: 471-6, 1995.
6. National Cholesterol Education Program (NCEP). The expert panel on blood cholesterol levels in children and adolescents. Pediatrics, 89(suppl. 3): 525-65, 1992.

7. Rabelo, L.M.; Fisberg, M. \& Martinez, T.L.R. Dislipidemia na infância e na adolescência. In: Carraza, F.R. \& Andriolo, A. Diagnóstico laboratorial em pediatria. 1.ed. São Paulo: Sarvier, 2000. p. 105-08.

8. Segundo Consenso Brasileiro sobre Dislipidemias. Detecção, avaliação e tratamento. Arq. Bras. Cardiol., 63(supl.): 1, 1996.

9. Segundo Consenso Brasileiro sobre Dislipidemias. Recomendações para o exame do perfil lipídico para clínicos e laboratórios. Atheros., 10(4): 109-20, 1999.

10. Widhalm, K.P. Paediatric guidelines for lipid reduction. Eur. Heart J., 8: 65-70, 1987.

11. Wissler, R.W. News insights into the pathogenes of atherosclerosis as revealed by PDAY. Atherosclerosis, 108(suppl.): S3-S20, 1994.
Endereço para correspondência Mario Seki Av. Bandeirantes, 310 CEP 86010-010 - Londrina-PR Tel.: (43) 322-3445 Fax: (43) 324-7510 e-mail: labimage@sercomtel.com.br 